\title{
Impact of reduced creatinine clearance on early heart transplantation outcomes: a propensity score adjusted analysis
}

\author{
Hrvoje Gašparović ${ }^{1 *}$, \\ Daniel Unić², \\ Lucija Svetina', \\ Jure Samardžić', \\ Maja Čikeš ${ }^{1}$, \\ Željko Baričević', \\ Boško Skorićc, \\ Tomislav Kopjar', \\ Darko Anić', \\ Višnja Ivančan', \\ Željko Sutlić ${ }^{2}$, \\ Bojan Biočina', \\ Davor Miličić \\ 'University of Zagreb School \\ of Medicine, University \\ Hospital Centre Zagreb, \\ Zagreb, Croatia \\ ${ }^{2}$ Clinical Hospital Dubrava, \\ Zagreb, Croatia
}

RECEIVED:

August 28, 2015 ACCEPTED: September 17, 2015

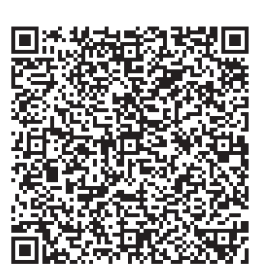

KEYWORDS: heart transplantation, renal failure, outcomes. CITATION: Cardiol Croat. 2015;10(9-10):208. | DOI: http://dx.doi.org/10.15836/ccar.2015.208 *ADDRESS FOR CORRESPONDENCE: Hrvoje Gašparović, Klinički bolnički centar Zagreb, Kišpatićeva 12, HR-10000 Zagreb, Croatia. / Phone: +385-1-2367-517 / E-mail: hgasparovic@gmail.com

ORCID: Hrvoje Gašparović, http://orcid.org/0000-0002-2492-3702 • Daniel Unić, http://orcid.org/0000-0003-2740-4067 Lucija Svetina, http://orcid.org/0000-0002-1242-3575 • Jure Samardžić, http://orcid.org/0000-0002-9346-6402 Maja Čikeš, http://orcid.org/0000-0002-4772-5549 • Željko Baričević, http://orcid.org/0000-0002-5420-2324 Boško Skorić, http://orcid.org/0000-0001-5979-2346• Tomislav Kopjar, http://orcid.org/0000-0002-4745-999X Darko Anić, http://orcid.org/0000-0002-7378-944X • Bojan Biočina, http://orcid.org/0000-0003-3362-9596 Davor Miličić, http://orcid.org/0000-0001-9101-1570

||||||||||||||||||||||||||||||||||||||||||||||||||||||||||||||||||||||||||||||||||||||||||||||||||||||||||||||||||||||||||||||||

GOAL: Renal insufficiency is a relative contraindication for isolated heart transplantation (HTx). ${ }^{\mathbf{1 - 3}} \mathrm{We}$ aimed to determine the independent effect of preoperative creatinine clearance ( $\mathrm{CrCl})$ on $\mathrm{HTx}$ outcomes.

PATIENTS AND METHODS: 220 patients underwent HTx in Croatia from 2008 to 2014 . Four patients were excluded due to missing data. Patients were dichotomized according to a $\mathrm{CrCl}$ cut-off value of $50 \mathrm{ml} /$

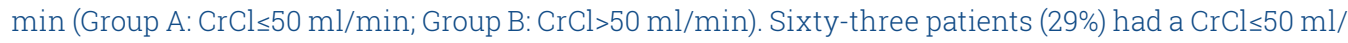
min. Propensity score (PS) adjustment was performed by accounting for recipient age and gender, AF, smoking, ischemic time, CPB time, BMI, mechanical circulatory assistance (MCS) and reoperation.

RESULTS: Patients in Group A were older ( $56 \pm 11$ vs. $49 \pm 12$ years, $P<0.001$ ) and had longer donor ischemic times (197 \pm 65 vs. $162 \pm 62 \mathrm{~min}, P<0.001)$. No significant differences were noted in recipient gender (48/63 (76\%) vs. $125 / 153$ (82\%) male, $P=0.355)$, donor age (39 \pm 13 vs. $39 \pm 12$ years, $P=0.875)$, donor gender (46/63

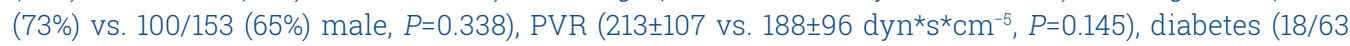
(29\%) vs. 34/153 (22\%), $P=0.382)$, reoperation (18/63 (29\%) vs. 34/153 (22\%), $P=0.382)$, CPB duration (175 \pm 62 vs. $158 \pm 56 \min , P=0.06)$ or preoperative MCS (6/63 (10\%) vs. 17/153 (11\%), $P=0.813)$. Six-month mortality was higher in patients with a $\mathrm{CrCl} \leq 50 \mathrm{ml} / \mathrm{min}$ (18/63 (29\%) vs. 19/153 (12\%); unadjusted OR 2.82 [95\% CI 1.36-5.84]; $P=0.009)$. Similarly, group A patients were more likely to require renal replacement therapy (RRT) (16/63 (25\%) vs. 17/153 (11\%); OR 2.72 (1.28-5.82); P=0.012). After PS adjustment these differences remained significant for both 6-month mortality and RRT (OR 2.44 [95\% CI 1.09-5.49]; P=0.030 and OR 3.36 [95\% CI 1.43-7.92]; $P=0.005$, respectively).

CONCLUSIONS: Patients with a $\mathrm{CrCl} \leq 50 \mathrm{ml} / \mathrm{min}$ undergoing isolated HTx had inferior 6-month survival and required RRT more commonly. The impact of $\mathrm{CrCl}$ remained significant after adjustment for multiple perioperative covariates. $\square$ Cardiologia Croatica 2015;10(9-10):208.

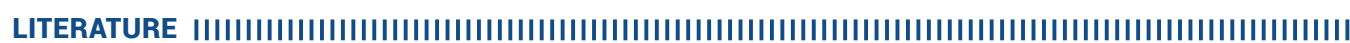

1. Ojo AO, Held PJ, Port FK, Wolfe RA, Leichtman AB, Young EW, et al. Chronic renal failure after transplantation of a nonrenal organ. N Engl J Med. 2003;349:931-40. DOI: http://dx.doi.org/10.1056/NEJMoa021744

2. Haglund NA, Feurer ID, Dwyer JP, Stulak JM, DiSalvo TG, Keebler ME, et al. Does renal dysfunction and method of bridging support in»uence heart transplant graft survival? Ann Thorac Surg. 2014;98:835-41. DOI: http://dx.doi.org/10.1016/j.athoracsur.2014.05.059

3. Gasparovic H, Milicic D, Biocina B. Contemporary challenges in the management of advanced heart failure. Croat Med J. 2014;55:551-2. DOI: http://dx.doi.org/10.3325/cmj.2014.55.551 